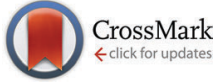

Cite this: Phys. Chem. Chem. Phys., 2015, 17, 365

\title{
The accurate calculation of the band gap of liquid water by means of GW corrections applied to plane-wave density functional theory molecular dynamics simulations $\dagger$
}

\author{
Changming Fang, ${ }^{* a}$ Wun-Fan Li, ${ }^{a}$ Rik S. Koster, ${ }^{a}$ Jiři Klimeš, ${ }^{b}$ Alfons van Blaaderen ${ }^{a}$ \\ and Marijn A. van Huis ${ }^{a}$
}

\begin{abstract}
Knowledge about the intrinsic electronic properties of water is imperative for understanding the behaviour of aqueous solutions that are used throughout biology, chemistry, physics, and industry. The calculation of the electronic band gap of liquids is challenging, because the most accurate ab initio approaches can be applied only to small numbers of atoms, while large numbers of atoms are required for having configurations that are representative of a liquid. Here we show that a high-accuracy value for the electronic band gap of water can be obtained by combining beyond-DFT methods and statistical time-averaging. Liquid water is simulated at $300 \mathrm{~K}$ using a plane-wave density functional theory molecular dynamics (PW-DFT-MD) simulation and a van der Waals density functional (optB88-vdW). After applying a self-consistent GW correction the band gap of liquid water at $300 \mathrm{~K}$ is calculated as $7.3 \mathrm{eV}$, in good agreement with recent experimental observations in the literature $(6.9 \mathrm{eV})$. For simulations of phase transformations and chemical reactions in water or aqueous solutions whereby an accurate description of the electronic structure is required, we suggest to use these advanced GW corrections in combination with the statistical analysis of quantum mechanical MD simulations.
\end{abstract}

Received 18th September 2014 Accepted 31st October 2014

DOI: $10.1039 / c 4 c p 04202 f$

www.rsc.org/pccp the same as those of ice, but with flexible bond-lengths and angles of $\mathrm{H}_{2} \mathrm{O}$ tetragons. ${ }^{3,5,6,12-14}$

To successfully perform simulations of chemical reactions in aqueous solutions, it is imperative that not only the atomic structure, but also the electronic structure is well described, whereby the electronic band gap can serve as the most important criterion. Many studies have already been performed on the electronic band gap of liquid water, but in the cases where accurate computational methods were used, these were applied to too few water molecules to be representative of liquid water. And in the cases where large numbers of water molecules were actually considered, methods such as cluster calculations were used which are known to be less accurate in predicting electronic properties.

Here we aim for high accuracy by applying $G W$ corrections to plane-wave density functional theory (PW-DFT) molecular dynamics (MD) simulations using a van der Waals density functional and a statistical analysis by the time-averaging of relevant physical parameters 'measured' during the MD simulations. We will now first discuss the experimental studies whereby the electronic structures of gas, water, and ice phases were investigated. ${ }^{2,15-25}$ Shibaguchi and co-workers investigated the electronic structure of ice by means of a combination of X-ray photoemission spectroscopy (XPS), Ultraviolet photoemission 
spectroscopy (UPS), and optical absorption spectroscopy, and obtained a band gap of $8.75 \mathrm{eV} .{ }^{17}$ Painter and co-workers measured the reflectance of liquid water between 1050 and $3000 \AA^{16}$ and found that the imaginary index of refraction starts at around $7.1 \mathrm{eV}$. From the measured real and imaginary parts of the index of refraction and dielectric constant, the authors suggested an exciton transition at $8.3 \mathrm{eV}$, an interband transition at $9.6 \mathrm{eV}$, and an electronic band gap of $9.0 \mathrm{eV}^{16}$ Other measurements of photoemission spectra provide a range of values for the band gap of water; $6.9 \mathrm{eV},{ }^{21,22} 7.0,{ }^{23} 8.7 \mathrm{eV},{ }^{24} 8.9 \mathrm{eV}^{25}$ and $9.0 \mathrm{eV} .^{16} \mathrm{As}$ reviewed by Winter and co-workers, such a variance in experimental data for the insulating ice phases and water is caused by many factors, such as electric charging, work functions, surface sensitivity (electron escape depths are typically $\sim 10-20 \AA ̊)$, vapour-solid or vapour-liquid mixing, impurities, etc. $^{2,19}$

Many theoretical and simulation studies have also been performed to study the structural and electronic properties of water phases. To study the structure and dynamics simple empirical potentials are widely used. ${ }^{26-30}$ Moreover, $a b$ initio Hartree-Fock (HF) and post-HF methods were employed to study water molecules and clusters, as well as ice phases. ${ }^{11,12,21,22,34-36}$ Finally, first-principles density functional theory (DFT) has also been widely used to study the structural and electronic properties of ice phases and liquid water. ${ }^{31-37}$

In theoretical studies of ice and liquid water, there are several issues to be noted. First, the standard DFT functionals, such as the local density approximation (LDA) and different GGA functionals, which are local or semi-local, do not describe accurately van der Waals interactions. This is an important issue since van der Waals interactions were shown to play an important role in water and ice phases, as well as other solids. ${ }^{39-50}$ For example, Santra and co-workers have recently investigated the ice phases at non-zero pressure using different van der Waals DFT exchange-correlation functionals and observed significant improvements of phase transition pressures of the ice phases compared to that of the PBE (after the names of authors, Perdew, Burke and Ernzerhof) functional. ${ }^{41,42}$ The van der Waals density functional was also found to describe water adsorption on metals well. ${ }^{43,44,49}$ Second, most of the calculations performed so far have focused on the description of the ground states of ice phases and water. However, experimental measurements of electronic properties, such as band gaps, are related to excitations of electrons. To describe the excitations another scheme needs to be used, such as the $G W$ approximation (whereby the self-energy is obtained from Green's function $G$ and the electronic screening effect $W$ ) which describes well the eigenstates of one-electron quasi-particle $(\mathrm{QP})$ levels which relate to electronic excitations. ${ }^{31,51-56}$ This method, but in a non-self-consistent $G 0 W 0$ fashion, was used by Hermann and co-workers to obtain the band gap of Ice-XI. ${ }^{31,32}$ Moreover, most first-principles molecular dynamics simulations employed supercells of 100 to 200 atoms, which can represent only a small number of molecules, and most simulations provide information about only one or a few configurations. This is not sufficient to obtain statistically meaningful results. ${ }^{56,57}$ Consideration of a large number of configurations with sufficient number of molecules to probe all relevant structures is needed. This approach was applied to analyse statistically the dominant defects in hydrogenated amorphous silicon nitrides covering different stoichiometries. ${ }^{51,57}$ The electronic structures were successfully simulated, including the energy gaps for the amorphous semiconductors with different types of defects. ${ }^{56,57}$

In the present manuscript, we report our results of a firstprinciples study on the $\mathrm{H}_{2} \mathrm{O}$ molecule, its dimer and Ice-XI, a low-temperature and low-pressure $\mathrm{H}_{2} \mathrm{O}$ phase with wellestablished $\mathrm{H}$ ordering ${ }^{8,10,11,26-32}$ as well as molecular dynamics simulations of water at $300 \mathrm{~K}$, using the van der Waals density functional developed by Dion $^{39}$ and implemented to the code VASP by Klimeš and co-workers. ${ }^{40}$ We also employ the selfconsistent GW0 approach $^{52,54}$ for Ice-XI. The almost linear relationship that was obtained between the QP (quasi-particle) energies and K-S (Kohn-Sham) eigenvalues is used to obtain the electronic structure including the band gap of water. Furthermore, we also analyse the charge distributions for $\mathrm{H}_{2} \mathrm{O}$ molecules, $\mathrm{H}_{2} \mathrm{O}$ dimers, and charge transfer between $\mathrm{H}_{2} \mathrm{O}$ molecules in water using the Bader charge approach ${ }^{58}$ which has been successfully applied to many molecules ${ }^{59}$ and crystalline solids. ${ }^{58,60}$ Distributions of charges at $\mathrm{O}$ and $\mathrm{H}$ were obtained for the simulated water, as well as for the isolated $\mathrm{H}_{2} \mathrm{O}$ monomer, its dimer and Ice-XI. The obtained electronic structures and the energy gaps for the ice phase and liquid water are in good agreement with available experimental data. This is not only useful for having a better understanding of the intrinsic properties of water, but also suggests that the same approach can be successfully applied to aqueous solutions that are widely used in various scientific disciplines and in industrial processes.

The paper is arranged as follows. In Section II, we introduce the (super)cells which were employed for the structural optimization of an isolated $\mathrm{H}_{2} \mathrm{O}$ molecule and its dimer, as well as of water with the experimental density at room temperature. The details of the computational methods are also described. The optimized structures of the isolated $\mathrm{H}_{2} \mathrm{O}$ molecule, its dimer and the lattice parameters of Ice-XI are presented in Section III.A. The structural and chemical bonding details and electronic properties of first-principles molecular dynamics simulated water are presented in Section III.B. The electronic properties of Ice-XI obtained using the DFT van der Waals density functional are discussed together with the GW0 corrections in Section III.C. In Section III.D, we discuss the electronic structure of water using the $G W$ corrections. The corrected curves of the density of states (DOS) are compared with the experimental measurements (mainly photoemission spectra). Finally a brief summary is presented in Section IV.

\section{Details of computational techniques}

For all the structural optimizations and total energy calculations of the isolated molecules and molecular dynamics simulations, we employed the Vienna code VASP (Vienna Ab initio Simulation Package $)^{57,58}$ which is based on the density functional theory (DFT) within the Projector-Augmented Wave (PAW) method. ${ }^{59,60}$ 
Dion's van der Waals density functional which includes nonlocal contributions was employed for the correlation energy term. ${ }^{39,40,44}$ The generalized gradient approximation ${ }^{61}$ (GGA) within the optB88 functional was employed for the exchange term. The electronic wave functions were sampled on dense grids $(20 \times 10 \times 10$ or 396 irreducible $k$-points $)$ in the Brillouin zone (BZ) of the crystals, using the Monkhorst and Pack method ${ }^{62}$ for the total energy and electronic structure calculations of liquids and solids. Structural optimizations were performed by the relaxation of both lattice parameters and coordinates of atoms for the crystals, while only atomic coordinates in a fixed cube were relaxed for the molecules.

It is noteworthy that water and the ice phase contain strong local bonds, $\mathrm{O} 2 \mathrm{p}-\mathrm{H} 1 \mathrm{~s}$, and therefore, the calculated valence electron total energy is expected to depend on the cut-off energies. We make a systematic investigation on the total energy calculations using different cut-off energies for the wave functions $\left(E_{\text {cut }}\right)$ and for the augmentation functions $\left(E_{\text {aug }}\right)$, which is about $30 \%$ higher than the corresponding $E_{\text {cut }}$ for a $\mathrm{H}_{2} \mathrm{O}$ molecule. The calculated results (see Fig. 1) show strong variations in the low energy range $\left(E_{\text {cut }} / E_{\text {aug }}=100 / 200-250 / 400 \mathrm{eV}\right)$ for the calculated bond-length, the $\mathrm{H}-\mathrm{O}-\mathrm{H}$ angle and the total valence electron energy. When $E_{\text {cut }} / E_{\text {aug }}$ is at least $300 / 450 \mathrm{eV}$, there is little dependence of the $\mathrm{O}-\mathrm{H}$ bond-length (within $0.001 \mathrm{~nm}$ ) and the $\mathrm{H}-\mathrm{O}-\mathrm{H}$ angle (within $0.1^{\circ}$ ) on the energy set, but still a weak dependence of the calculated total energy on the energy set. The calculated total valence electron energy becomes stable when the cut-off energy is over $650 \mathrm{eV}$ (within $1 \mathrm{meV}$ per atom). Therefore, to ensure cut-off independent results in all total energy calculations the cut-off energy of the

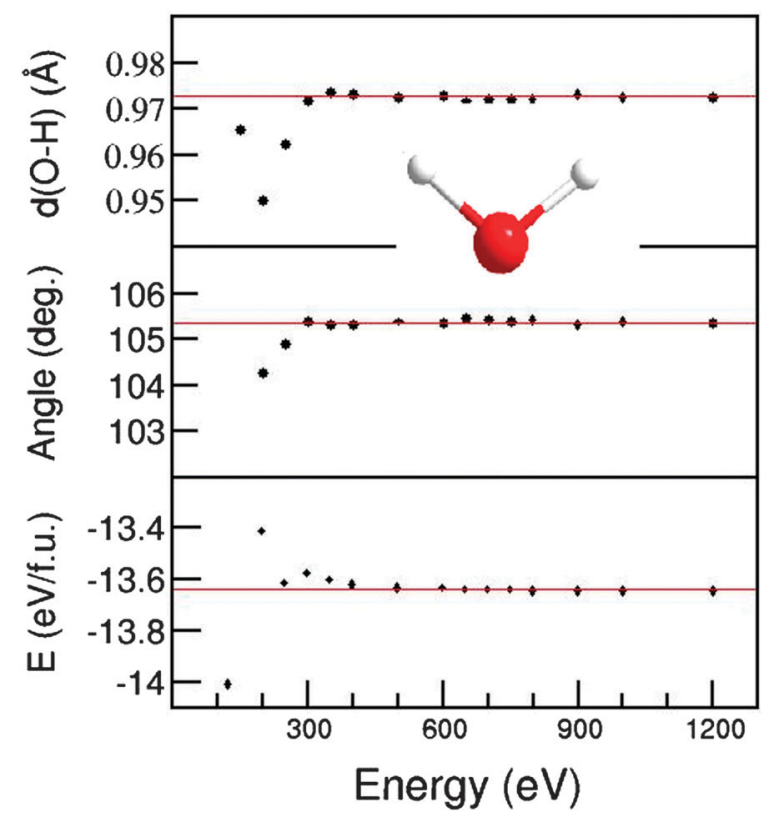

Fig. 1 Convergence of the $\mathrm{O}-\mathrm{H}$ bond-length (top), the $\mathrm{H}-\mathrm{O}-\mathrm{H}$ angle (middle) and the calculated total energy (bottom) as a function of the cutoff energy for an isolated $\mathrm{H}_{2} \mathrm{O}$ molecule (the schematic structure being the inset) using the van der Waals density functional. The red solid lines indicate the converged values. wave functions was set to be $750 \mathrm{eV}$, and the cut-off energy of the augmentation functions was set to be $1000 \mathrm{eV}$. The low sensitivity of the $\mathrm{H}-\mathrm{O}-\mathrm{H}$ angle and the $\mathrm{O}-\mathrm{H}$ bond-length on the cut-off energy also allows us to use lower cut-off energies for the molecular-dynamics simulations. We used a cut-off energy of $320 \mathrm{eV}$, and only the $\Gamma$ point in the Brillouin zone (BZ) in all molecular-dynamics simulations for the sake of finding a reasonable balance between computational feasibility and the accuracy of the results since the cell is large.

\section{Calculated results and discussion}

III.A. Structures of the $\mathrm{H}_{2} \mathrm{O}$ molecule, the $\mathrm{H}_{2} \mathrm{O}$ dimer, and the Ice-XI phase

We first discuss the properties of a water monomer, a dimer, and Ice-XI. Tables 1-4 list the calculated results for an isolated $\mathrm{H}_{2} \mathrm{O}$ molecule and its dimer (Fig. 2b), as well as the Ice-XI phase with ordered hydrogen arrangements ${ }^{11,12,26}$ (see Fig. 2a). The calculated results are compared with recent theoretical studies. ${ }^{11,33,34,38,47,63,67}$

The $\mathrm{O}-\mathrm{H}$ bond-length in the $\mathrm{H}_{2} \mathrm{O}$ monomer is $0.973 \AA$, close to the experimental values within $0.02 \AA .{ }^{64}$ There are many theoretical studies using various functionals, including the 'hybrid' B3LYP method which adopted an all-electron $6311 \mathrm{G}(\mathrm{p}, \mathrm{d})$ basis set, ${ }^{11,12}$ X3LYP which contains van der Waals interactions, ${ }^{35,35}$ and the ab initio Hartree-Fock method within

Table 1 Calculated results for an isolated $\mathrm{H}_{2} \mathrm{O}$ molecule using the optB88-vdW functional compared with the former theoretical studies and experimental observations

\begin{tabular}{|c|c|c|c|}
\hline & Methods & $d(\mathrm{O}-\mathrm{H})(\AA)$ & $\begin{array}{l}\text { Angle } \\
\mathrm{H}-\mathrm{O}-\mathrm{H}\left({ }^{\circ}\right)\end{array}$ \\
\hline This work & optB88-vdW & 0.973 & 105.40 \\
\hline \multirow{3}{*}{$\mathrm{Xu}$ and Goddard ${ }^{35,36}$} & $\mathrm{HF}$ & 0.941 & 106.2 \\
\hline & PBEPBE & 0.971 & 104.0 \\
\hline & X3LYP & 0.961 & 105.1 \\
\hline \multirow[t]{3}{*}{ Casassa, et al. ${ }^{11,12}$} & HF-CRY-c & 0.940 & 106.2 \\
\hline & B3LYP-CRY-c & 0.961 & 105.1 \\
\hline & PW91-CRY-c & 0.968 & 104.3 \\
\hline Silvestralli + Parrinello ${ }^{34}$ & GGA-BLYP & 0.972 & 104.4 \\
\hline Jurečka ${ }^{38}$ & HF-CCSDT & 0.959 & 104.2 \\
\hline Exp. $^{15}$ & & 0.957 & 104.5 \\
\hline Exp. ${ }^{16}$ & & 0.958 & 104.45 \\
\hline
\end{tabular}

Table 2 Calculated results for an $\mathrm{H}_{2} \mathrm{O}$ dimer using the optB88-vdW functional compared with the former theoretical studies and experimental observations

\begin{tabular}{|c|c|c|c|c|}
\hline & Methods & $\begin{array}{l}d(\mathrm{O}-\mathrm{H})(\AA) \\
\text { range }\end{array}$ & $d(\mathrm{O}-\mathrm{O})(\AA)$ & $\begin{array}{l}E_{\text {form }} \\
\text { (eV/f.u.) }\end{array}$ \\
\hline This work & optB88-vdW & $0.97 / 0.98$ & 2.866 & -0.236 \\
\hline \multirow{3}{*}{$\mathrm{Xu}$ and Goddard ${ }^{35,36}$} & $\mathrm{HF}$ & $0.941 / 0.945$ & 3.048 & -0.16 \\
\hline & PBEPBE & $0.970 / 0.981$ & 2.899 & -0.28 \\
\hline & X3LYP & $0.957 / 0.968$ & 2.908 & -0.24 \\
\hline \multirow[t]{3}{*}{ Casassa, et al..$^{11,12}$} & HF-CRY-c & $0.944^{a}$ & 3.042 & -0.12 \\
\hline & B3LYP-CRY-c & 0.969 & 2.925 & -0.21 \\
\hline & PW91-CRY-c & 0.979 & 2.880 & -0.24 \\
\hline Jurečka ${ }^{38}$ & HF-CCSDT & & & -0.218 \\
\hline Exp. $^{12,17}$ & & & 2.976 & -0.24 \\
\hline
\end{tabular}

${ }^{a}$ The $\mathrm{O}-\mathrm{H}$ distance with hydrogen bonding with another $\mathrm{O}$. 
Table 3 Calculated results for the Ice-XI phase

\begin{tabular}{|c|c|c|}
\hline Method & $\begin{array}{l}\text { Lattice pars. }(\AA), \\
V\left(\AA^{3} / \text { f.u. }\right)\end{array}$ & Atomic coordinates \\
\hline optB88-vdW & $a=4.343(-3.5 \%)$ & $\mathrm{O} 14 \mathrm{a}(0,0.6502,0.0586)$ \\
\hline \multirow[t]{4}{*}{ (this work) } & $b=7.609(-2.5 \%)$ & O2 4a(0,0.3190,0.9358) \\
\hline & $c=7.035(-4.0 \%)$ & H1 $4 \mathrm{a}(0,0.6571,0.2017)$ \\
\hline & $V=29.06(-9.6 \%)$ & H2 4a(0,0.5226,0.0197) \\
\hline & & H3 8b $(0.6870,0.7526,0.9811)$ \\
\hline \multirow{4}{*}{ HF-CRY $^{11}$} & $a=4.57(+1.5 \%)$ & \\
\hline & $b=8.40(+7.7 \%)$ & \\
\hline & $c=7.69(+4.9)$ & \\
\hline & $V=36.85(14.6 \%)$ & \\
\hline \multirow[t]{4}{*}{ B3LYP ${ }^{11}$} & $a=4.42(-1.8 \%)$ & \\
\hline & $b=7.85(0.7 \%)$ & \\
\hline & $c=7.23(-1.3)$ & \\
\hline & $V=31.36(-2.5 \%)$ & \\
\hline \multirow{5}{*}{ diffraction ${ }^{65}$} & $a=4.5019$ & O1 $4 \mathrm{a}(0,0.6648,0.0631)$ \\
\hline & $b=7.7978$ & $\mathrm{O} 24 \mathrm{a}(0,0.3255,0.9360)$ \\
\hline & $c=7.3280$ & H1 4a $(0,0.6636,0.1963)$ \\
\hline & $V=32.16$ & H2 4a(0,0.5363,0.0183) \\
\hline & & H3 8b $(0.6766,0.7748,0.9817)$ \\
\hline
\end{tabular}

Table 4 The Bader charge distributions (in units of e) on the $\mathrm{O}, \mathrm{H}$ atoms in the isolated $\mathrm{H}_{2} \mathrm{O}$ monomer, a dimer, and Ice-XI

\begin{tabular}{lll}
\hline Structure & $\mathrm{O}$ & $\mathrm{H}$ \\
\hline Monomer & -1.28 & 0.64 \\
Dimer & -1.18 to -1.19 & 0.55 to 0.61 \\
Ice_XI & $-1.30,-1.31$ & 0.64 to 0.67
\end{tabular}
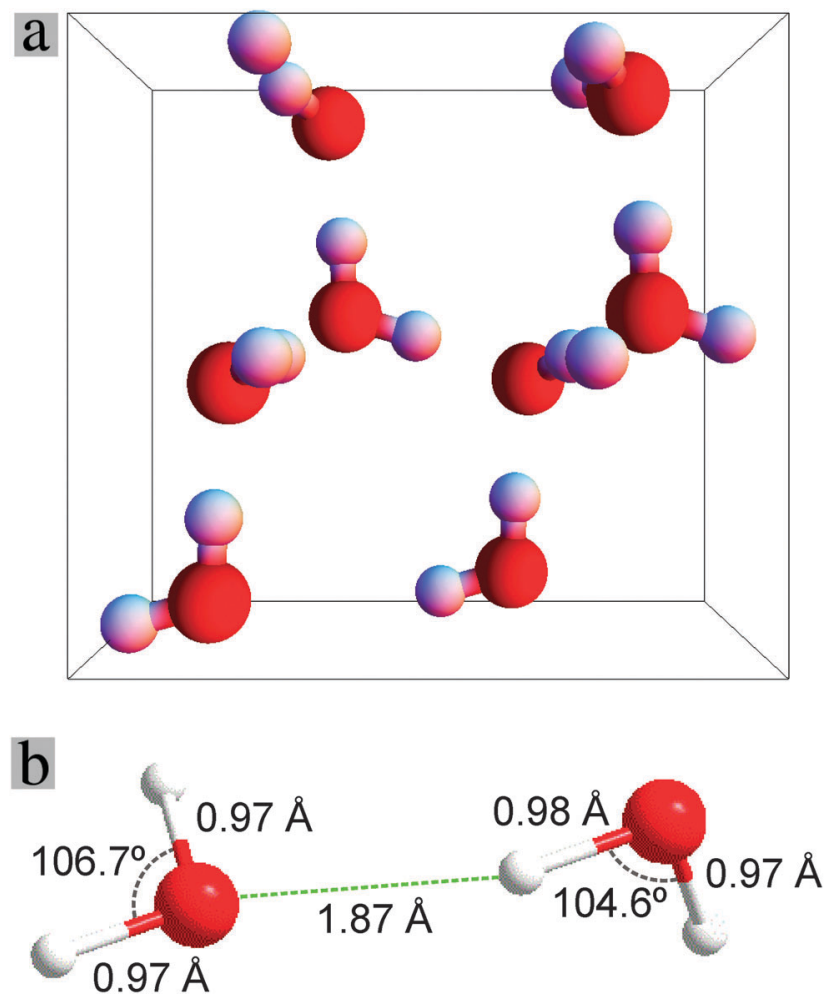

Fig. 2 (a) Schematic structure of ice Ice-XI with the projection along the [001] direction. (b) Structure of $\mathrm{a} \mathrm{H}_{2} \mathrm{O}$ dimer, the unit for the angles is degree, for the $\mathrm{O}-\mathrm{H}$ and $\mathrm{O} \cdots \mathrm{H}$ bonds (bond lengths in $\AA$ ). coupled-cluster singles, doubles, triples (HF-CCSDT) wave function. ${ }^{38}$ Here we compare our results with a few of them (Table 1). The obtained $\mathrm{O}-\mathrm{H}$ bond is close to those obtained by the $a b$ initio HF methods and by first-principles DFT approximations (Table 1). The presently calculated $\mathrm{H}-\mathrm{O}-\mathrm{H}$ angle is just about $1.0^{\circ}$ larger than the measured one, and in good agreement with former theoretical calculations, as shown in Table 1.

Table 2 and Fig. $2 \mathrm{~b}$ also show the calculated results for the $\mathrm{H}_{2} \mathrm{O}$ dimer. The calculated bonds and angles are in line with the former calculations. The calculated $\mathrm{O}-\mathrm{H}$ bond for the $\mathrm{H}$ participating in the hydrogen bond $(0.98 \AA)$ is slightly longer than the other 3 short $\mathrm{O}-\mathrm{H}$ bonds $(0.97 \AA)$. This difference (0.01 ̊) was also obtained by other DFT and HF calculations. ${ }^{11,33,34,38}$ The calculated binding energy is close to the experimental value, with slight overestimation, as observed previously. ${ }^{12,27}$

The ground state of $\mathrm{H}_{2} \mathrm{O}$ is the Ice-XI phase..$^{9-12,26-30,67-69}$ The crystal structure of Ice-XI is orthorhombic with space group $\mathrm{Cmc2}_{1}$ (nr. 36). The structure at $5 \mathrm{~K}$ was determined by Leadbetter and co-workers using neutron diffraction (Table 3$)^{69}$ and investigated theoretically by several groups using different approaches. . $^{11,26,27,32,41,42}$

We performed full structural optimization of this ice phase using the van der Waals density functional optB88-vdW. The results are shown in Table 3. It is clear that our calculated lattice parameters are $2.5-4.0 \%$ smaller than the corresponding experimental values. That difference is similar to the PW91 results obtained by Casassa and co-workers. ${ }^{11,12}$ We also note that HF calculations overestimate the volume while better agreement with the experiment can be obtained with much more computationally expensive correlated methods, such as the Random Phase Approximation. ${ }^{47}$

The Bader charge analysis ${ }^{58}$ shows that there is charge transfer of 0.64 electrons per hydrogen atom from $\mathrm{H}$ to $\mathrm{O}$. In the $\mathrm{H}_{2} \mathrm{O}$ dimer, the charges are almost the same for the two $\mathrm{O}$ ions ( -1.18 electrons), which is about 0.10 electrons less than that of an isolated molecule, in agreement with former calculations. ${ }^{58}$ There are also some differences between the charges on the $\mathrm{H}$ atoms: the $\mathrm{H}$ of the long $\mathrm{O}-\mathrm{H}$ bond has a charge of +0.55 electrons, which is smaller than the charges (about 0.60 electrons) on the other three $\mathrm{H}$ atoms. For the ice phase, the charge on the two oxygen ions is about -1.30 electrons and the $\mathrm{H}$ ions are charged in the range of $0.64-0.67$ electrons, as shown in Table 4.

\section{III.B. Charges in water at $300 \mathrm{~K}$}

The thermal history of water simulated at $300 \mathrm{~K}$ is shown in Fig. 3 which also includes the variation of the cohesive energy for the water system on time at $300 \mathrm{~K}$. It is clearly shown that the energy lowers down with time and reaches the equilibrium after about $3.5 \mathrm{ps}$ (or 7000 iterations).

First we briefly discuss the structure of the simulated water. We monitored the processes of the molecular dynamics simulations. We observed no sign of charged ions/clusters, neither a single proton $\left(\mathrm{H}^{+}\right)$or $\left(\mathrm{H}_{3} \mathrm{O}\right)^{+},\left(\mathrm{H}_{5} \mathrm{O}_{2}\right)^{+}$, nor $(\mathrm{OH})^{-}$or $\left(\mathrm{H}_{3} \mathrm{O}_{2}\right)^{-}$or 


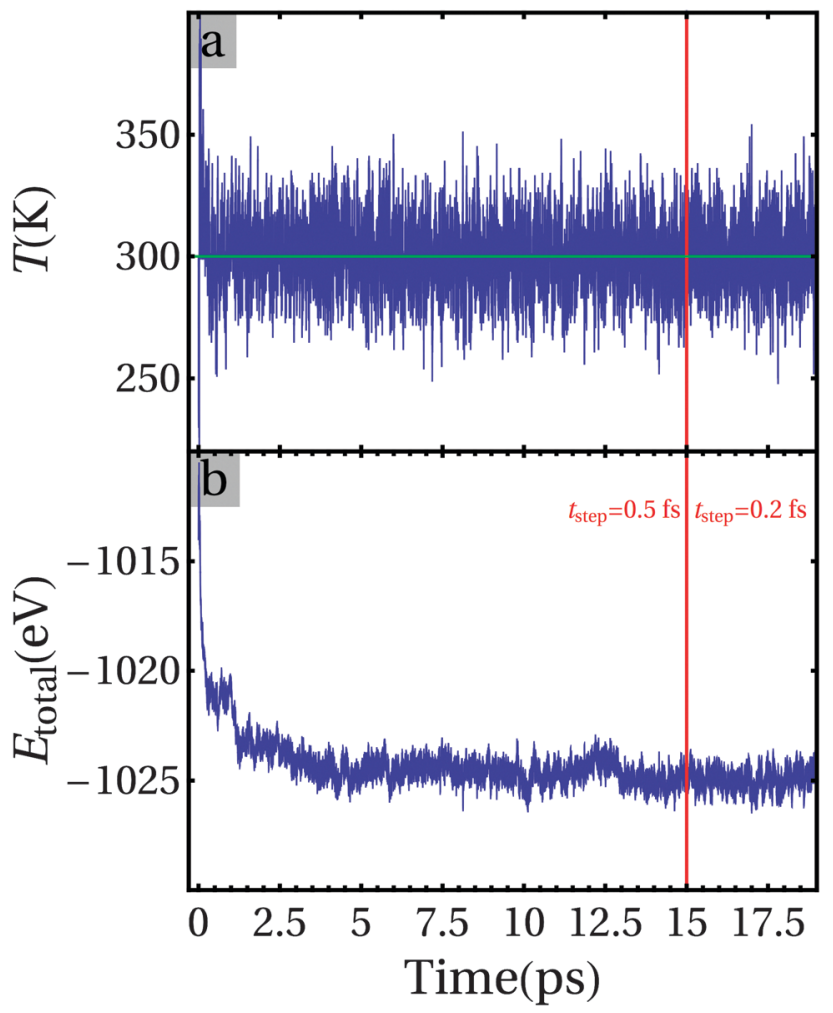

Fig. 3 The thermal history of temperature (a) and total valence electron energy (b) of water simulated at a temperature of $300 \mathrm{~K}$.

$\left(\mathrm{H}_{3} \mathrm{O}_{2}\right)^{-}$clusters. This is understandable considering the high formation energies of such ions or charged clusters., ${ }^{2,6,22}$ In addition, the Born-Oppenheimer approximation was used in our molecular dynamics simulations, so that proton tunnelling is neglected. However, we observed a $2\left(\mathrm{H}_{2} \mathrm{O}\right)$ cluster with a very short intermolecular $(\mathrm{O}-\mathrm{H})$ bond of $1.4 \AA$ (Fig. S1, ESI $\dagger$ ), which exists for about 50 fs. Fig. 4a shows a typical configuration of water obtained from the first-principles molecular dynamics simulations at $300 \mathrm{~K}$. We analysed several configurations obtained for each of about 0.5 ps time-gap. The $\mathrm{O}-\mathrm{H}$ bonds distributions in $\mathrm{H}_{2} \mathrm{O}$ molecules together with the Bader charge distributions are shown in Fig. S2 (ESI $\dagger$ ). Apparently there is a strong similarity among the distributions of the intra-molecule bonds for the different configurations (snapshots at different times) with significant deviations from a regular Gaussian-type distribution. This is also true for the Bader charge distributions (Fig. S2, ESI $\dagger$ ).

As discussed previously, compared with the size of a drop of water $(\sim \mu \mathrm{m})$ the small number of water molecules in the cell $\left(64 \mathrm{H}_{2} \mathrm{O}\right.$ molecules, 192 atoms, size $\left.\sim \mathrm{nm}\right)$ can on the one hand be regarded as being local. The fluctuations of bonding in time, on the other hand, reflect the stochastic nature of liquid water. Therefore, time-averaging can and should be used to determine the physical properties of water. Here we simulate these properties by time-averaging over 2 ps.

The results are shown in Fig. 4 and Fig. S2 (ESI $\dagger$ ). It is clear that the time-averaged distributions show more or less regular Gaussiantype distributions, in comparison to the large scattering observed
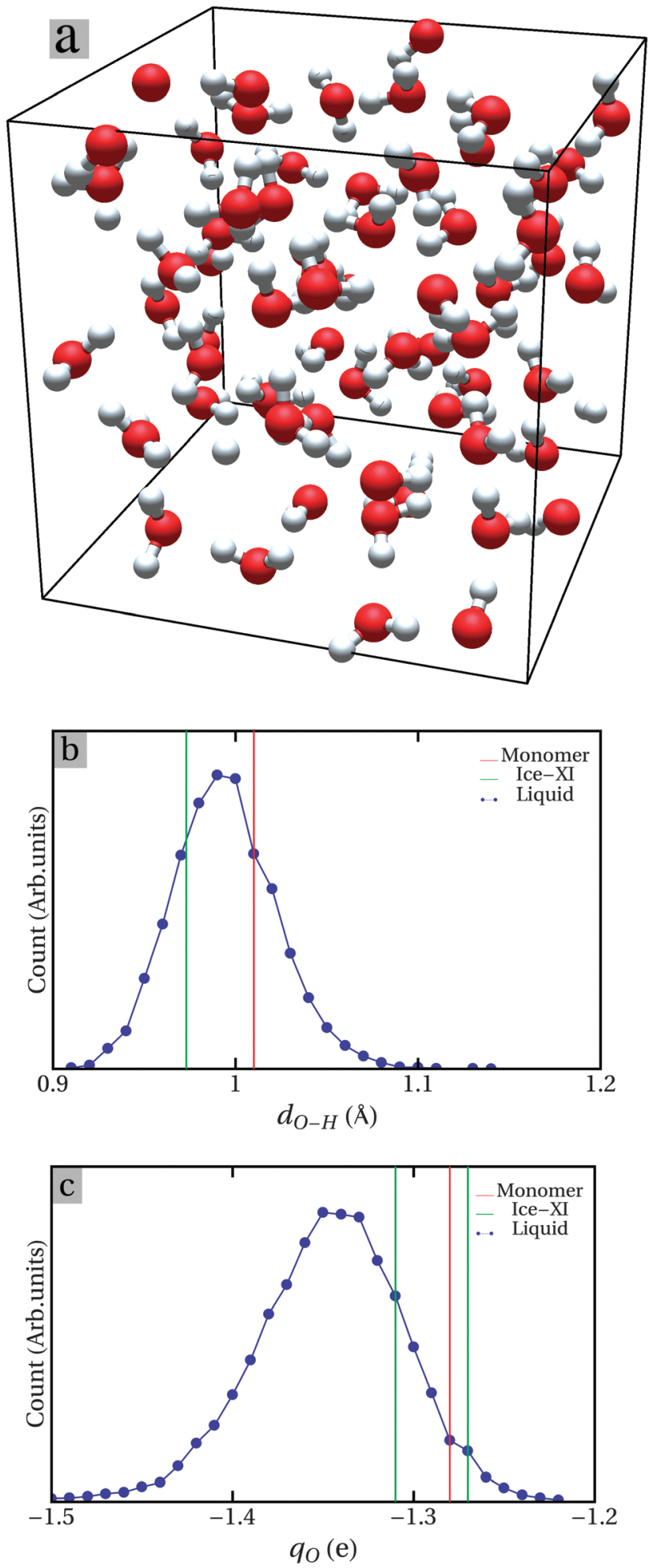

Fig. 4 Snapshot of one typical water structure during the simulations at $300 \mathrm{~K} \mathrm{(a)}$, the distributions of $\mathrm{O}-\mathrm{H}$ bond lengths (b) and the Bader charge of $\mathrm{O}$ ions (c) time-averaged over a period of 2 ps during the simulation of water at $300 \mathrm{~K}$ (about 100 samples).

between individual snapshot configurations (Fig. S2, ESI $\dagger$ ). The $\mathrm{O}-\mathrm{H}$ bond-lengths in water range from 0.92 to $1.08 \AA$ with the width of a half-peak height of $0.1 \AA$, centred at $0.99 \AA$, which is between that of 
the isolated $\mathrm{H}_{2} \mathrm{O}$ monomer $(0.97 \AA)$ and that of the Ice-XI phase (1.01 ̊). This corresponds to the model of Bernal and co-workers. ${ }^{3-5}$ As shown in Fig. 4c, Bader's charge at $\mathrm{O}$ sites of water is between -1.22 e (electrons) and -1.48 e with a broad peak at about -1.34 e. The averaged value (about $1.34 \mathrm{e}$ ) of the charges at $\mathrm{O}$ ions is slightly larger than those of an isolated $\mathrm{H}_{2} \mathrm{O}$ monomer and Ice-XI, although the averaged $\mathrm{O}-\mathrm{H}$ bond-lengths of water are close to those of the monomer and ice. This can be understood from the Brown bond theory ${ }^{66,67}$ where the valence $\left(V_{i}\right)$ of the central atom to the $i$ th neighbour is defined as

$$
V_{i}=\exp \left[-\left(R_{i}-R_{0}\right) / A\right]
$$

where $R_{i}$ is the observed bond length, $R_{0}$ is a tabulated parameter expressing the (ideal) bond length when element $i$ has exactly valence 1 , and $A$ is a universal empirical constant, typically $0.37 \AA$. For example, a bond of $0.1 \AA$ shorter gives a $V$ value of 1.31 Valence Unit (VU) while a bond of $0.1 \AA$ longer gives a $V$ value of $0.76 \mathrm{VU}$. Considering the strong similarity between charge transfer and the valence of atoms/ions, the higher values of charge transfer in water is understandable.

\section{III.C. Electronic structure of Ice-XI and water at $300 \mathrm{~K}$}

To have an overview of the electronic properties of different phases of water, the eigenvalues of an isolated $\mathrm{H}_{2} \mathrm{O}$ molecule (5a), its dimer (5b), the total density of states for Ice-XI (5c), and one snapshot of liquid water $(5 \mathrm{~d})$ are displayed in Fig. 5.

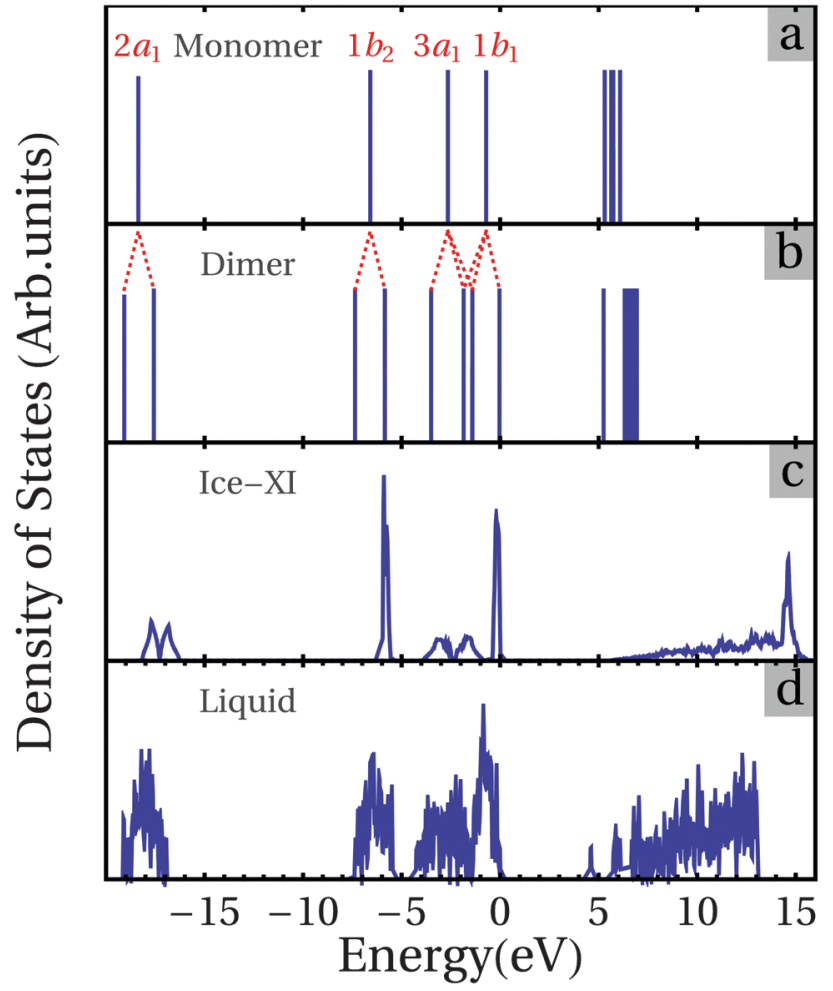

Fig. 5 The DFT-van der Waals density functional calculations of eigenenergies of an isolated $\mathrm{H}_{2} \mathrm{O}$, and its dimer; the tDOS of the ice phase and a snapshot of water simulated at $300 \mathrm{~K}$ with notable localization of electronic states.
Fig. 6 shows the calculated partial and total density of states (pDOS and tDOS) (6a) and the corresponding dispersion curves along the high symmetry lines of the Brillouin Zone (BZ) for the orthorhombic Ice-XI phase using Dion's DFT-v/d Waals density functional. ${ }^{39,40}$ The eigenvalues of the isolated $\mathrm{H}_{2} \mathrm{O}$ molecule originate from the interactions between $\mathrm{O} 2 \mathrm{~s}, \mathrm{O} 2 \mathrm{p}$, and $\mathrm{H} 1 \mathrm{~s}$ states. ${ }^{15-19,33,73}$ There are early assignments for the four occupied eigenstates, $2 \mathrm{a}_{1}$ (O $2 \mathrm{~s}$ with some $\mathrm{H} 1 \mathrm{~s}$ character), $1 \mathrm{~b}_{2}$ and $3 \mathrm{a}_{1}$ (O 2p hybridized with $\mathrm{H}$ 1s), $1 \mathrm{~b}_{1}$ (non-bonding $\mathrm{O} 2 \mathrm{p}$ ), and the lowest unoccupied $4 \mathrm{a}_{1}(\mathrm{O} 2 \mathrm{~s} 2 \mathrm{p}$ and $\mathrm{H} 1 \mathrm{~s})$ and $2 \mathrm{~b}_{2}$ (O $2 \mathrm{p}$ and $\mathrm{H} 1 \mathrm{~s}$ ) states. $2 \mathrm{a}_{1}, 1 \mathrm{~b}_{2}$ and $3 \mathrm{a}_{1}$ belong to bonding states while $4 \mathrm{a}_{1}$ and $2 \mathrm{~b}_{2}$ have anti-bonding characters.
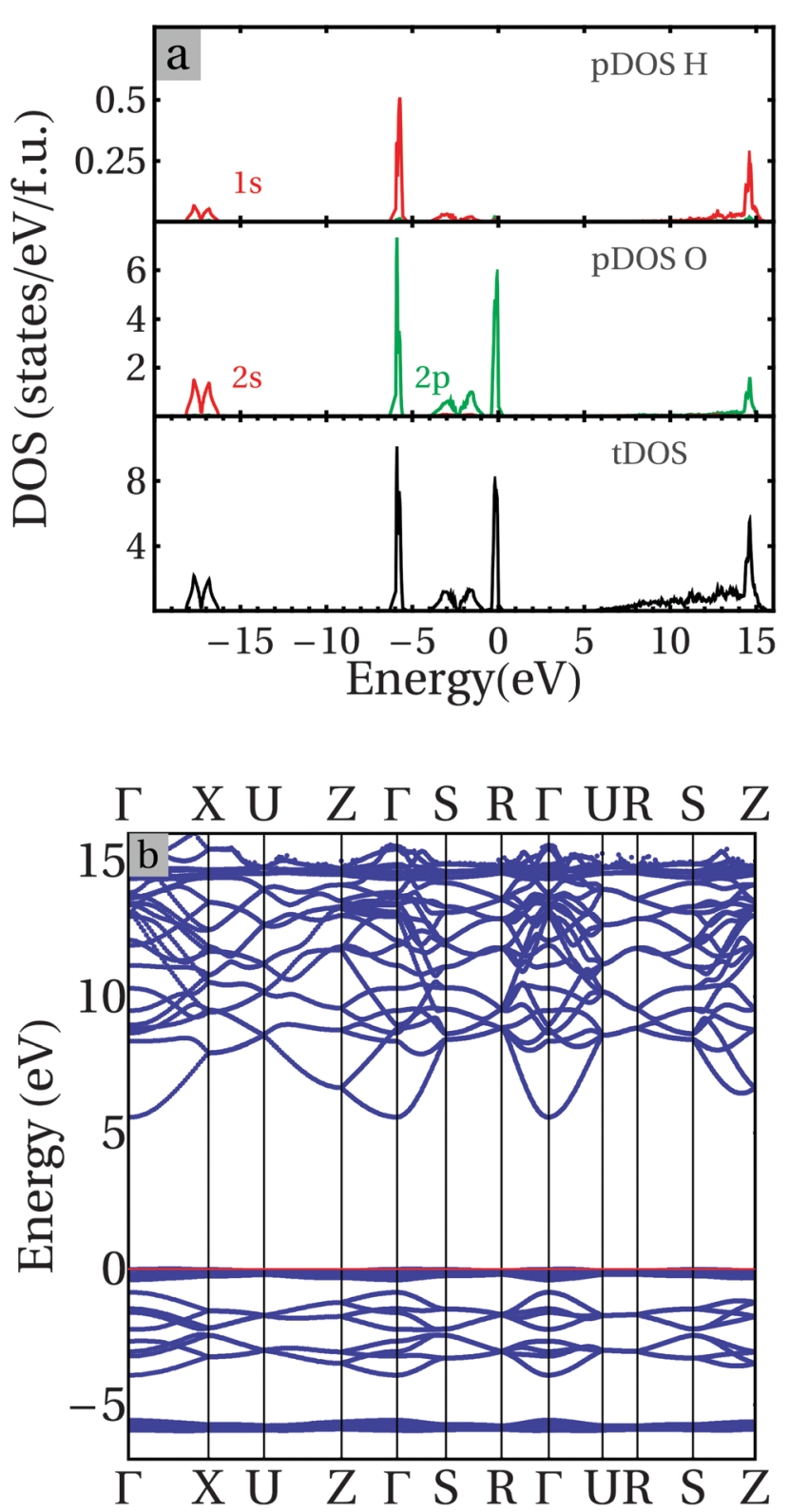

Fig. 6 (a) Partial densities of states (pDOS), (b) dispersion curves along the high symmetry lines in the Brillouin zone (BZ) for the ground state of the orthorhombic Ice-XI phase. The Fermi level is set to be the top of the valence band. 
For the $\mathrm{H}_{2} \mathrm{O}$ dimer, the distortion of the molecules and the interactions between the molecules (Fig. 2) cause splitting (about $1.5 \mathrm{eV}$ ) of the $1 \mathrm{~b}_{2}$ states, and further hybridizations between $3 a_{1}$ and $1 b_{1}$ states that induce the lowering of new $3 \mathrm{a}_{1}$ and shifting up of new $1 \mathrm{~b}_{1}$, together with the creation of two new hybridized states (bonding $3 \mathrm{a}_{1} / 1 \mathrm{~b}_{1}$ and anti-bonding $\left(3 a_{1} / 1 b_{1}\right)^{*}$ states $)$. The lowest unoccupied states $4 a_{1}$ are also lowered in energy due to further hybridization. Clearly the energy gap between the occupied and unoccupied states is strongly reduced in comparison to that of the $\mathrm{H}_{2} \mathrm{O}$ molecule.

Fig. 5 also includes the tDOS for Ice-XI for the sake of comparison. Overall the results for the Ice-XI phase agree well with the early symmetry study by Parravicini and Resca who used the Bloch sums formed by $\mathrm{H}_{2} \mathrm{O}$ molecule orbitals as an expansion set for cubic ice, ${ }^{68,72}$ and the recent DFT-GGA calculations by Prendergast and co-workers for the Ice-Ih phase ${ }^{34}$ considering the strong similarities between the different $\mathrm{H}_{2} \mathrm{O}$ ordering of these phases. ${ }^{3-6,10-12}$ Here we briefly discuss the electronic structure of Ice-XI, with the partial DOS and dispersion curves along the high symmetry lines in Fig. 6. There are five separated bands. We use here the labels used in the early assignments ${ }^{15,16,73}$ to designate the bands. Two of them, band $1 b_{2}$ at $-6 \mathrm{eV}$, and band $1 b_{1}$ at the top of the valence band (set to $0 \mathrm{eV}$ ), show an atomic-orbital behavior with very small band widths. The lowest one $\left(2 \mathrm{a}_{1}\right)$ belongs to the $\mathrm{O} 2 \mathrm{~s}$ states hybridized with $\mathrm{H}$ 1s orbitals with two peaks at about $-17.7 \mathrm{eV}$ and $-16.8 \mathrm{eV}$, respectively. The width of the $\mathrm{O} 2 \mathrm{~s}$ bands is about $2 \mathrm{eV}$, with the Fermi level being set at the top of the valence band. This indicates strong interactions between the $\mathrm{O} 2 \mathrm{~s}$ and $\mathrm{H} 1 \mathrm{~s}$ states. However, they do not contribute to the chemical bonding since both bonding and antibonding states are fully occupied.

There is a gap of about $10.4 \mathrm{eV}$ between the $\mathrm{O} 2 \mathrm{~s}$ band $\left(2 \mathrm{a}_{1}\right)$ and the $\mathrm{O} 2 \mathrm{p}\left(1 \mathrm{~b}_{2}\right)$ states with a significant hybridization with $\mathrm{H}$ $1 \mathrm{~s}$ states. The very narrow band indicates a strongly localized character of the $\mathrm{O} 2 \mathrm{p}-\mathrm{H} 1 \mathrm{~s}$ bonding corresponding to direct bonding. The next band with two peaks at about $-3.2 \mathrm{eV}$ (bonding $3 \mathrm{a}_{1}$ ) and $-1.6 \mathrm{eV}$ (anti-bonding $3 \mathrm{a}_{1}{ }^{*}$ ) is dominated by $\mathrm{O} 2 \mathrm{p}$ states mixed with some $\mathrm{H} 1$ s character. The top valence band belongs to the non-bonding $\mathrm{O} 2 \mathrm{p}$ states $\left(1 \mathrm{~b}_{1}\right)$. This band is very narrow and shows a strong localized character in nature. The lower part of the conduction band (from about 5.6 to about $+14.5 \mathrm{eV}$ ) is dominated by $\mathrm{O} 2 \mathrm{p} / 3$ s states mixed with some $\mathrm{H} 1 \mathrm{~s}$ character. These states in the lower conduction band show significant dispersion over $5 \mathrm{eV}$. There is a peak at about $14.5 \mathrm{eV}$ dominated by $\mathrm{O} 2 \mathrm{p}-\mathrm{H}$ 1s states (anti-bonding, $4 \mathrm{a}_{1}$ ).

The van der Waals density functional produced an energy gap of $5.55 \mathrm{eV}$. The top of the valence band is at $(1 / 4,0,0)$ in the Brillouin Zone while the bottom of the conduction band is at $\Gamma(0,0,0)$. The direct energy gap at $\Gamma$ is $5.58 \mathrm{eV}$, just slightly larger than the indirect gap. The calculated energy gap is smaller than the experimental value of $8.75 \mathrm{eV},{ }^{17}$ which is not surprising since DFT functionals are known to underestimate the energy gaps of insulators and semiconductors. ${ }^{52,72}$

We return to Fig. 5 for the electronic structure of a snapshot of water at $300 \mathrm{~K}$. Though similar to that of the Ice-XI phase, the tDOS curves of water at $300 \mathrm{~K}$ are more dispersed: there are localized states with tailed states near the band gap. As mentioned before, the present cell containing $64 \mathrm{H}_{2} \mathrm{O}$ molecules can be regarded as a representative of a small fraction of a real system. Therefore, to have a better understanding of an amorphous or a liquid system simulated with a limited number of atoms, statistics are required based on a number of samples, rather than based on one or a few samples, to produce physically meaningful results. ${ }^{32,54,72}$ Water is an insulator with strong local distortions, though topologically similar to that of ice phases. ${ }^{1-3}$ Correspondingly the DOS curve is composed of very localized states (Fig. 5). As shown in Fig. 4b there are broad $\mathrm{O}-\mathrm{H}$ bonds and the distributions of charges at $\mathrm{O}$ (Fig. 4c) and at $\mathrm{H}$ (Fig. S2, ESI $\dagger$ ) in water. It is also notable that although there

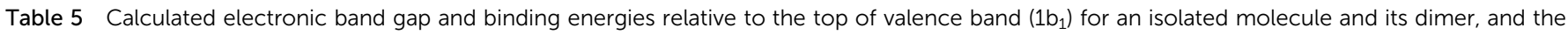

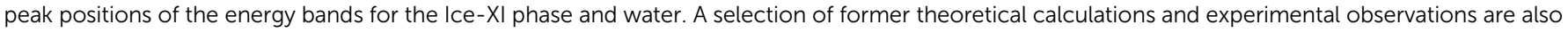
listed for the sake of comparison

\begin{tabular}{|c|c|c|c|c|c|c|}
\hline & Author year & Method & $\left(3 \mathrm{a}_{1} / 3 \mathrm{a}_{1}^{*}\right)^{b}-1 \mathrm{~b}_{1}$ & $\left(1 b_{2} / 1 b_{2}^{*}\right)^{b}-1 b_{1}$ & $\left(2 \mathrm{a}_{1}-2 \mathrm{a}_{1}^{*}\right)^{b}-1 \mathrm{~b}_{1}$ & $\Delta E_{\text {gap }}$ \\
\hline \multirow[t]{3}{*}{$\mathrm{H}_{2} \mathrm{O}$ mono. } & This work & optB88-vdW & $1.94^{a}$ & $5.88^{a}$ & $17.64^{a}$ & 6.02 \\
\hline & Couto 2005 & GGA_MPW1 & $2.1^{33} /$ & $6.0^{33}$ & & $12.4^{33}$ \\
\hline & Banna 1986 & Exp. & $2.20^{71}$ & $6.18^{71}$ & $20.02^{71}$ & \\
\hline \multirow[t]{3}{*}{$\mathrm{H}_{2} \mathrm{O}$ dimer } & This work & optB88-vdW & $3.47 / 1.82 / 1.38$ & $7.33 / 5.82$ & $19.04 / 17.54$ & 5.29 \\
\hline & Couto 2005 & GGA_MPW1 & $3.7 / 2.1 / 1.4^{33}$ & $7.5 / 6.1^{33}$ & $23.0 / 21.3^{33}$ & $11.33^{33}$ \\
\hline & & $\mathrm{HF}$ & $3.5 / 2.0 / 1.3^{33}$ & $7.3 / 6.1^{33}$ & $24.6 / 23.2^{33}$ & $13.97^{33}$ \\
\hline \multirow{4}{*}{ Ice-XI } & This work & optB88-vdW & $(2.99 / 1.47)$ & 5.60 & 17.06 & 5.55 \\
\hline & & $G W 0$ & $(3.36 / 1.65)$ & 6.29 & 18.20 & 9.17 \\
\hline & Banna 1986 & Exp. & $1.9^{71}$ & $5.3^{71}$ & $18.7^{71}$ & $8.75^{71}$ \\
\hline & Nordlund 2008 & Exp. & $(2.40 / 1.80)^{19}$ & $6.04^{19}$ & & \\
\hline \multirow[t]{7}{*}{ Liquid } & This work & optB88-vdW & 1.9 & 5.7 & 17.3 & $\sim 3.9$ \\
\hline & & S_GW0 & 2.1 & 6.4 & 19.5 & $\sim 7.3$ \\
\hline & Shibaguchi 1977 & Exp. & $2.35^{17}$ & $6.18^{17}$ & $19.40^{17}$ & \\
\hline & Banna 1986 & Exp. & $2.2^{71}$ & $6.4^{71}$ & $20.0^{71}$ & \\
\hline & Winter 2004 & Exp & $2.34^{19}$ & $6.38^{19}$ & $19.74^{19}$ & \\
\hline & Nordlund 2008 & Exp & $2.50^{20}$ & $6.37^{20}$ & & \\
\hline & Coe 1997, 2001 & Exp. & & & & $6.9^{21,22}$ \\
\hline
\end{tabular}

${ }^{a}$ There is only one peak for each of the $2 \mathrm{a}_{1}, 1 \mathrm{a}_{2}$ and $3 \mathrm{a}_{1}$ states for one $\mathrm{H}_{2} \mathrm{O}$ molecule (Fig. 5). ${ }^{b}$ The states are overlapped for liquid water. 
is a short hydrogen bond in the $2\left(\mathrm{H}_{2} \mathrm{O}\right)$ clustering (Fig. S1, ESI $\dagger$ ), this clustering contributes nothing to the top of the valence band and the bottom of the conduction band, as shown in Fig. S5 (ESI $\dagger$ ).

That is understandable since the electronic structure of water is determined mainly by the short $\mathrm{O}-\mathrm{H}$ bonds in the water molecule. In fact our analysis showed a change of the Fermi level in a range of about $0.9 \mathrm{eV}$ for water in 2 ps (Fig. S3, ESI $\dagger$ ). Meanwhile, as shown in Fig. S3 (ESI $\dagger$ ), the lowest unoccupied eigen-energies vary, albeit only slightly $(0.1 \mathrm{eV})$. The major change of the DOS comes from the top of valence states due to the wide local bonding. Therefore, the integrated tDOS can be considered as a representative of the electronic properties of water as a whole (Fig. 5). Clearly the integrated tDOS have broader bandwidths for each band. There are tailed states at the edges of the valence band and conduction bands. This results in a band gap of about $3.9 \mathrm{eV}$ for the present calculations, largely due to the tailed states at the top of the valence band. The overall electronic characteristics are shown in Table 5 .

\section{III.D. GW0 corrections and comparison with experimental observations}

It is clear from Table 5 that there are many differences between the calculated electronic properties of the ice phase and water and the experimental photoemission spectra (PES) measurements. This is due to the fact that the DFT approaches describe well the ground states of electronic properties of materials, while most experimental methods, e.g. PES, are sensitive to the excitation of electrons. The $G W$ method, a beyond-DFT approach, is very suitable to describe the excitation processes of sp electrons (a quasi-particle (QP) approach) and has been successfully applied to many systems. ${ }^{49,50,52,53,70}$ For example, Hermann and co-workers performed a non-self-consistent G0W0 correction for ice. ${ }^{31,32}$ The requirements for computational capability limit us to the Ice-XI phase. Fig. 7 shows the results of partially self-consistent GW0 corrections for Ice-XI.

The calculations show that the direct $G W 0$ correction has already improved the K-S energy gap (DFT-PBE) significantly. About 3 iterations are required to reach convergence (Fig. 7a). Fig. 7b shows that there is almost linear relationship between the QP energies and the Kohn-Sham (KS) eigenvalues for the sp electrons in Ice-XI. The first itineration $(G 0 \mathrm{~W} 0)$ provided an energy gap of about $8.66 \mathrm{eV}$ which is strongly enhanced as compared to that $(5.55 \mathrm{eV})$ from the van der Waals density functional theory. This $G 0 W 0$ value is also close to that (about $8.8 \mathrm{eV}$ ) obtained by Hermann and co-workers. ${ }^{31,32}$ Furthermore, the QP energy gap from the $G W 0$ correction is about $9.2 \mathrm{eV}$, considerably larger than that of the KS energy gap, as shown in Table 5. This obtained QP band gap is close to the experimental value, $8.75 \mathrm{eV}^{71}$

Considering the topological similarity between the regular structure of the ice phase and water, it is reasonable to use the obtained linear relationships between the QP energies and KS eigenvalues of Ice-XI as a correction to the electronic structure of water. Fig. 8 shows the obtained tDOS curves for simulated
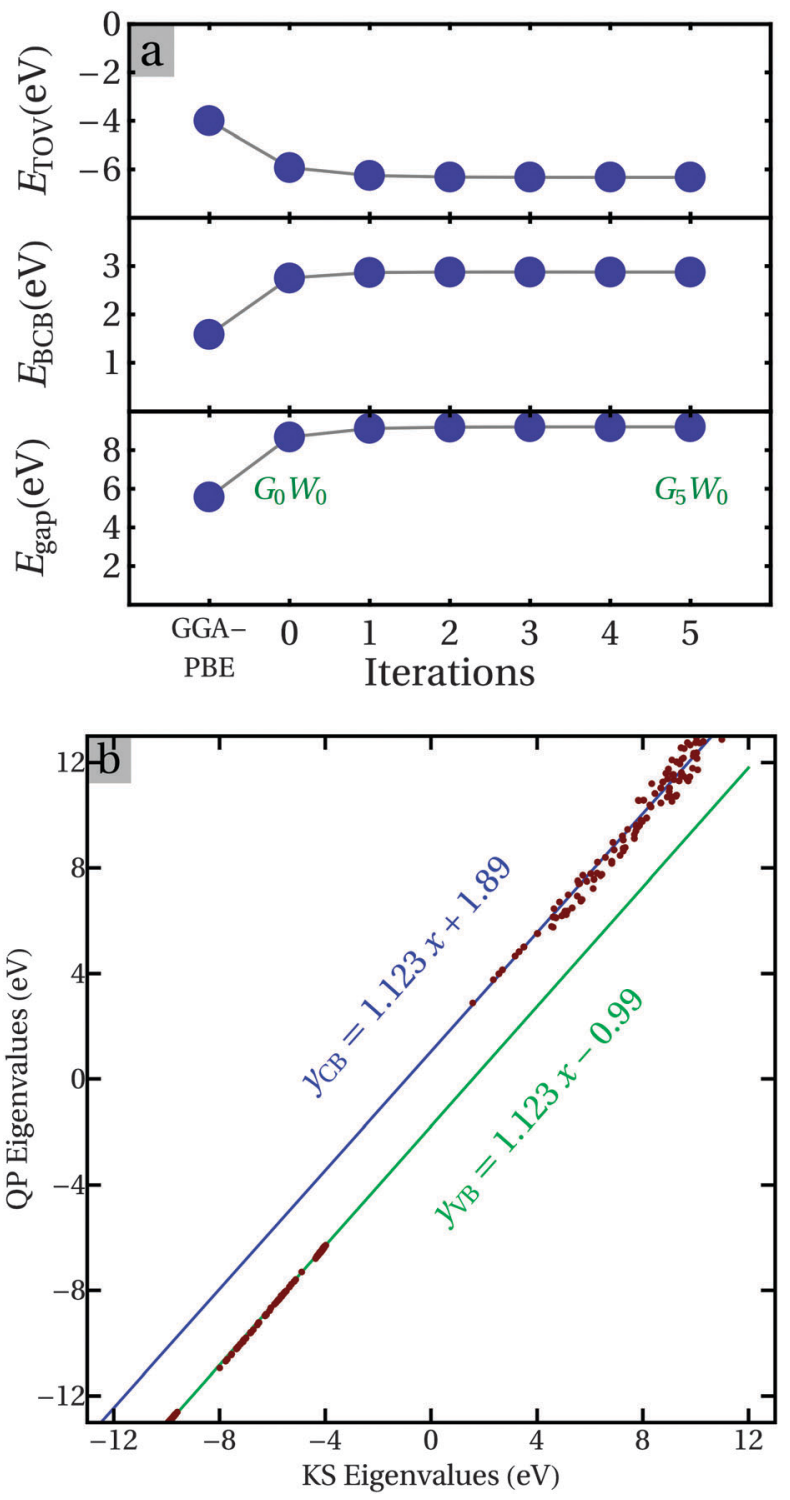

Fig. 7 Convergence of the partially self-consist GWO calculations (a), and relationships between the DFT-GGA K-S eigenvalues and GWO QP energies (b). The green and blues lines represent the fitting functions.

water in comparison with the PES spectra. ${ }^{19,20,75}$ Table 5 lists the obtained electronic characteristics of the eigen-energies of water and the ice phase. It is clear that the obtained vdW-DFT tDOS curves have the peak mismatch with the experimental observations.

This can be understood from the fact that standard DFT provides the electronic properties of a solid at its ground state while the PES measurements are related to excitons of electrons, as mentioned before. There is excellent agreement between valence bands of the QP tDOS and the PES observations (also in Fig. 8). The peaks of the hybridized $\mathrm{O} 2 \mathrm{~s}$ and $\mathrm{H} 1 \mathrm{a}$ states $\left(2 \mathrm{a}_{1}\right)$ match well with the PES measurements (not shown in Fig. 8). Naturally, the heights/intensities of the peaks are dependent on the cross-sections of the photons with the characteristics of electrons, but the positions of the peaks can be easily recognized 


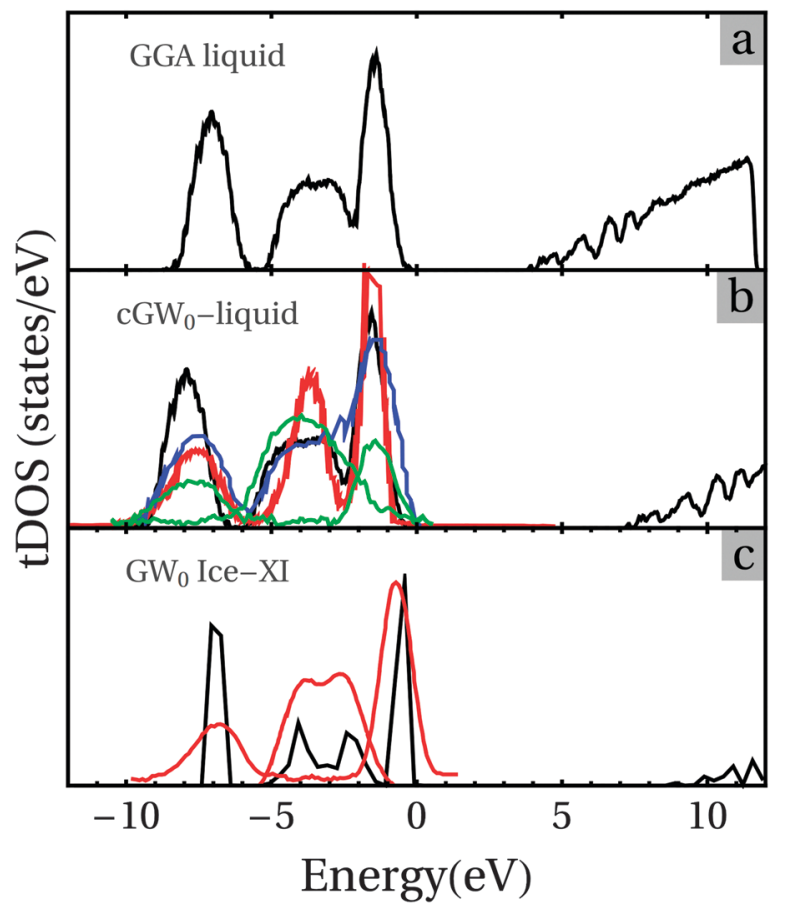

Fig. 8 The time-averaged tDOS curves obtained by the optB88-vdW calculations (a) and its QP tDOS (b) of water with the GW-correction using the relationships between the DFT KS-eigenvalues and the GWO QP energies (see Fig. $6 \mathrm{~b}$ and the corresponding text). The GWO tDOS curve for the Ice-XI phase is shown in figure (c) for the sake of comparison. The Fermi level for all the curves is set to be the top of the valence bands. The colored curves in (b) and (c) are experimental measurements: in (c) the red curve for ice, ${ }^{20}$ in (b) for water from ref. 75 (red line), from ref. 19 (blue line) and from ref. 20 (green lines). The black line represents the calculated results using the GWO corrections.

and are listed in Table 5. The obtained energy gap for the integrated tDOS of water at $300 \mathrm{~K}$ is about $7.3 \mathrm{eV}$ for the QP, which is much higher than that of the KS gap (about 3.9 eV). The QP gap of 7.3 eV is in between the experimental values ranging from about 6.9 to $9.0 \mathrm{eV}$. The energy gap found in the present work is slightly larger than the predicted values (6.01 to $6.55 \mathrm{eV}$ ) obtained from a combination of configuration interaction (CI) and time-dependent DFT (TD-DFT) calculations. ${ }^{76,77}$ In particular, the QP gap is in very good agreement with the recent experimental measurements of the band gap of $6.9 \mathrm{eV} .^{21,22}$

\section{Conclusions}

Using the first-principles van der Waals density functional, we performed structural optimizations for the isolated $\mathrm{H}_{2} \mathrm{O}$ monomer, its dimer, and the crystalline ice phase. The obtained results agree well with the available experimental values and former theoretical calculations. First-principles molecular-dynamics simulations for water at $300 \mathrm{~K}$ showed the high stability of the structural element $\mathrm{H}_{2} \mathrm{O}$, whereby configurations of the $\mathrm{H}_{2} \mathrm{O}$ elements retain tetragonal coordination with distortions. The Bader charge analysis showed that water is strongly ionic with a charge transfer of $0.64 e / \mathrm{H}$ to $\mathrm{O}$.
To describe the electronic structure and in particular the electronic band gap of liquid water with high accuracy, we have employed the $G W$ correction based on the relationship between the Kohn-Sham (KS) eigenvalues and the quasi-particle (QP) eigenvalues of the ice phase. Moreover, we have taken about one hundred snapshot configurations of liquid water with 192 atoms in the simulation cell, and performed statistical time averaging to obtain a more reliable estimate of the physical properties. Overall, the self-consistent $G W 0$ corrections produce electronic structures and electronic band gaps of both Ice-XI and water, which are in excellent agreement with experimental observations. The present knowledge is helpful in understanding many processes and reactions in water and aqueous solutions, such as sol-gel silica processes. ${ }^{74,78} \mathrm{We}$ suggest this approach as an accurate method to investigate the electronic properties of aqueous solutions and chemical reactions therein.

\section{Acknowledgements}

We thank Dr Bing Liu (UU, NL) for useful discussions. $\mathrm{MvH}$ acknowledges a VIDI grant from the Dutch Science Foundation NWO. JK was supported by the Austrian Science Fund (FWF) within the SFB ViCoM (Grant F 41).

\section{References}

1 V. Bartels-Rausch, J. H. E. Bergeron, R. Cartwright, J. L. Escribano, H. Finney, P. J. Grothe, J. Gutijerrez, W. F. Haapala, J. B. C. Kuhs, S. D. Pettersson, C. I. Price, D. J. Sainz-Diaz, G. Stokes, E. S. Strazzulla, H. Thomson, H. Trinks and N. Uras-Aytemiz, Rev. Mod. Phys., 2012, 84, 885 .

2 B. Winter and M. Fauber, Chem. Rev., 2006, 106, 1176-1211.

3 J. D. Bernal, Proc. R. Soc. London, Ser. A, 1958, 247, 421.

4 J. D. Bernal, Proc. Phys. Soc. A, 1949, 62, 537.

5 R. H. Fowler and J. D. Bernal, Trans. Faraday Soc., 1933, 29, 1049-1056.

6 J. D. Bernal and R. H. Fowler, J. Chem. Phys., 1933, 1, 515.

7 B. Q. Christner, C. E. Morris, C. M. Foreman, R. M. Cai and D. C. Sands, Science, 2008, 319(5867), 1214.

8 H. Iglev, M. Schmeisser, K. Simeonidis, A. Thaller and A. Laubereau, Nature, 2006, 439(7073), 183-186.

9 B. J. Murray, D. A. Knopf and A. K. Bertram, Nature, 2006, 434(7030), 202-205.

10 Y. Fang, B. Xiao, J. M. Tao, J. W. Sun and J. P. Perdew, Phys. Rev. B, 2013, 87, 214101.

11 S. Casassa, M. Calatayud, K. Doll, C. Minot and C. Pisani, Chem. Phys. Lett., 2005, 409, 110.

12 C. Vega, J. L. F. Abascal and P. G. Debenedetti, Phys. Chem. Chem. Phys., 2011, 13, 19660.

13 J. L. Finney, J. Phys.: Conf. Ser., 2007, 57, 40-52.

14 J. R. Errington and P. G. Debenedetti, Nature, 2001, 409, 318-321. 
15 R. E. Verrall and W. A. Senior, J. Chem. Phys., 1968, 50, 2746.

16 L. R. Painter, R. N. Hamm, E. T. Arakawa and R. D. Birkhoff, Phys. Rev. Lett., 1968, 21, 282-284.

17 T. Shibaguchi, H. Onuki and R. Onanka, J. Phys. Soc. Jpn., 1977, 42, 152-158.

18 N. Timneanu, C. Caleman, J. Hajdu and D. van der Spoel, Chem. Phys., 2004, 299, 277.

19 B. Winter, R. Weber, W. Widdra, M. Dittmar, M. Fauber and I. V. Hertel, J. Phys. Chem. A, 2004, 108, 2625.

20 D. Nordlund, M. Odelius, H. Bluhm, H. Ogasawara and L. G. W. Pettersson, Chem. Phys. Lett., 2008, 460, 86.

21 J. V. Coe, A. D. Earhart, M. C. Cohen, G. J. Hoffman, H. W. Sarkas and K. H. Bowen, J. Chem. Phys., 1997, 107, 6023.

22 J. V. Coe, Int. Rev. Phys. Chem., 2001, 20, 33-58.

23 D. Grand, A. Bernas and E. Amouyal, Chem. Phys., 1979, 44, 73.

24 A. Bernas, C. Ferradini and J.-P. Jay-Gerin, Chem. Phys., 1997, 222, 151.

25 T. Goulet, A. Bernas, C. Ferradini and J.-P. Jay-Gerin, Chem. Phys. Lett., 1990, 170, 492.

26 G. T. Barkema and J. de Boer, J. Chem. Phys., 1993, 99, 2059.

27 G. T. Barkema and M. W. J. Newman, Phys. Rev. E, 1998, 57, 1155.

28 H. Itoh, K. Kawamura, T. Hondoh and S. Mae, J. Chem. Phys., 1998, 109, 4894.

29 N. Guisoni and V. B. Henriques, J. Chem. Phys., 2001, $115,5238$.

30 R. Ramirez, N. Neuerburg and C. P. Herro, J. Chem. Phys., 2012, 137, 134503.

31 A. Hermann and P. Schwerdtfeger, Phys. Rev. Lett., 2011, 106, 187403.

32 A. Hermann, N. W. Ashcroft and R. Hoffmann, Proc. Natl. Acad. Sci. U. S. A., 2012, 109, 745.

33 P. Cabral do Couto, S. G. Estácio and B. J. Costa Cabral, J. Chem. Phys., 2005, 123, 054510.

34 P. L. Silvestrelli and M. Parrinello, J. Chem. Phys., 1999, 111, 3572.

$35 \mathrm{X} . \mathrm{Xu}$ and W. A. Goddard III, J. Chem. Phys., 2004, 121, 4068.

$36 \mathrm{X} . \mathrm{Xu}$ and A. Goddard III, J. Phys. Chem. A, 2004, 108, 2305.

37 D. Nordlund, H. Ogasawar, K. J. Andersson, M. Tatarkhanov, M. Salmerón, L. G. M. Petterson and A. Nilson, Phys. Chem. Chem. Phys., 2006, 8, 1985-1993.

38 P. Jurečka, J. Šponer, J. Černy and P. Hobza, Phys. Chem. Chem. Phys., 2006, 8, 1985-1993.

39 M. Dion, H. Rydberg, E. Schröder, D. C. Langreth and B. I. Lundqvist, Phys. Rev. Lett., 2004, 92, 246401.

40 J. Klimeš, D. R. Bowler and A. Michelides, J. Phys.: Condens. Matter, 2010, 22, 022201.

41 B. Santra, J. Klimeš, D. Alfe, A. Tkatchenko, B. Slater, A. Michaelides, R. Car and M. Scheffler, Phys. Rev. Lett., 2011, 107, 185701.
42 B. Santra, J. Klimeš, D. Alfe, A. Tkatchenko, B. Slater, A. Michaelides, R. Car and M. Scheffler, J. Chem. Phys., 2013, 139, 154702.

43 J. Carrasco, J. Klimeš and A. Michaelides, J. Chem. Phys., 2013, 138, 024708.

44 G. Román-Peréz and J. M. Soler, Phys. Rev. Lett., 2009, 103, 096102.

45 G. Graziano, J. Klimeš, F. Fernandez-Alonso and A. Michaelides, J. Phys.: Condens. Matter, 2012, 24, 424216.

46 J. Klimě̌ and A. Michaelides, J. Chem. Phys., 2012, 137, 120901.

47 M. Macher, J. Klimeš, C. Franchini and G. Kresse, J. Chem. Phys., 2014, 140, 084502.

48 R. G. Parr, Annu. Rev. Phys. Chem., 1983, 34, 631.

49 V. G. Ruiz, W. Liu, E. Zojer, M. Scheffler and A. Tkatchenko, Phys. Rev. Lett., 2012, 108, 146103.

50 P. Rinke, A. Qteish, J. Neugebauer, C. Feysoldt and M. Schffler, New J. Phys., 2006, 7, 126.

51 G. Kresse, M. Marsman, L. E. Hintzsche and E. Flage-Larsen, Phys. Rev. B, 2012, 85, 045205.

52 L. E. Hintzsche, C. M. Fang, T. Watts, M. Marsman, G. Jordan, M. W. P. E. Lamers, A. W. Weeber and G. Kresse, Phys. Rev. B, 2012, 86, 235204.

53 E. Flage-Larsen, O. M. Løvvik, C. M. Fang and G. Kresse, Phys. Rev. B, 2013, 88, 165310.

54 R. F. W. Bader, T. T. Nguyen and Y. Tal, Rep. Prog. Phys., 1981, 44, 893.

55 C. M. Fang, M. A. Van Huis, J. Jansen and H. W. Zandbergen, Phys. Rev. B, 2011, 84, 094102.

56 C. M. Fang, M. H. F. Sluiter, M. A. van Huis and H. W. Zandbergen, Phys. Rev. B, 2012, 86, 134114.

57 G. Kresse and J. Hafner, Phys. Rev. B, 1994, 49, 14251-14269.

58 G. Kresse and J. Hafner, Phys. Rev. B, 1994, 49, 14251.

59 P. E. Blöchl, Phys. Rev. B, 1994, 50, 17953-17979.

60 G. Kresse and J. Furthmüller, Phys. Rev. B, 1999, 54, 11169.

61 J. P. Perdew, K. Burke and M. Ernzerhof, Phys. Rev. Lett., 1996, 77, 3865-3868.

62 H. J. Monkhorst and J. D. Pack, Phys. Rev. B, 1976, 13, 5188.

63 J. C. G. Pereira, C. R. A. Catlow, G. D. Price and R. M. Almeida, J. Sol-Gel Sci. Technol., 1997, 8, 55.

64 L. A. Curtiss and J. A. Pople, J. Mol. Spectrosc., 1975, 55, 1. 65 A. J. Leadbetter, R. C. Ward, J. W. Clark, P. A. Tucker, T. Matsuo and H. Suga, J. Chem. Phys., 1985, 82, 424.

66 I. D. Brown, The Chemical Bond in Inorganic Chemistry. IUCr Monographs in Crystallography 12, Oxford Science Publications, Oxford University Press, Oxford, U.K., 2002.

67 I. D. Brown, Chem. Rev., 2009, 109, 6858-6919.

68 G. P. Parravicini and L. Resca, Phys. Rev. B, 1973, 8, 3009.

69 W. A. Goddard III and W. J. Hunt, Chem. Phys. Lett., 1974, 24, 464 .

70 L. E. Hintzsche, C. M. Fang, M. Marsman, G. Jordan, M. W. P. E. Lamers, A. W. Weeber and G. Kresse, Phys. Rev. B, 2013, 88, 155204. 
71 M. S. Banna, B. H. McQuaide, R. Malutzki and V. Schmidt, 75 K. C. Chen, T. Tsuchiya and J. D. Mackenzie, J. Non-Cryst. J. Chem. Phys., 1986, 84, 4739. Solids, 1986, 81, 227.

72 M. E. Tuckerman, D. Marx and M. Parrinello, Nature, 2002, 76 G. Brancat, N. Rega and V. Barone, Phys. Rev. Lett., 2008, 417, 925. 100, 107401.

73 A. Hassanali, M. K. Prakash, H. Eshet and M. Parrinello, 77 P. C. do Couto and B. J. Costa Cabral, J. Chem. Phys., 2007, Proc. Natl. Acad. Sci. U. S. A., 2011, 108, 20410. 126, 014509.

74 T. T. Trinh, A. P. J. Jansen, R. A. Van Santen and E. J. Meijer, 78 F. F. Xia, D. W. Zeng, H. B. Yi and C. H. Fang, J. Phys. Chem. A, J. Phys. Chem. C, 2009, 113, 2647. 2013, 117, 8468. 